\title{
On the cohomology ring of some homogeneous spaces.
}

\author{
By
}

\author{
Hirosi TODA
}

(Received, March 20, 1974)

\section{Introduction.}

Let $G$ be a compact connected Lie group and let $U$ be its torsion free connected subgroup of maximal rank. The purpose of the present paper is to establish a method to describe the integral cohomology ring $H^{*}(G / U)$, by a minimum system of generators and relations, from the results of the $\bmod p$ cohomology ring $H^{*}\left(G ; Z_{p}\right)$ of $G$ and the rational cohomology $\operatorname{ring} H^{*}(G / U ; Q)$ of $G / U$.

The homogeneous space $G / U$ is equivalent to the total space of a principal $G$-bundle over the classifying space $B U$ of $U$. In $\S 1$, we shall discuss mod $p$ cohomology of principal $G$-bundles of such type, and the result will be stated in Theorem 1.1. A description of the integral cohomology ring $H^{*}(G / U)$ will be given in Theorem 2.1 of $\S 2$, and will be exhibited for simple $G$ and $U=T$, a maximal torus of $G$, in $\S 3$ as applications. Another application to the cohomology structure of $G$ will be seen in forthcoming papers.

\section{$\S 1$ Mod p cohomology of some principal bundles.}

Let $G$ be a compact connected Lie group and consider a principal $G$-bundle 


$$
G \stackrel{i}{\longrightarrow} X \stackrel{\pi}{\longrightarrow} B .
$$

We always assume that the base space $B$ is arcwise connected and its cohomology groups are finitely generated for each dimension. So, the same holds for the total space $X$.

Let $p$ be a prime and consider the following three hypotheses: (1.2) The cup-product gives an isomorphism

$$
\Delta\left(x_{1}, \ldots, x_{m}\right) \otimes M \cong H^{*}\left(G ; Z_{p}\right)
$$

for a graded submodule $M=\sum M^{j}$ and homogeneous elements $x_{i}(i=1, \ldots$, $m)$, where $\Delta\left(x_{1}, \ldots, x_{m}\right)$ indicates the submodule spanned by the monomials $\left\{x_{1}^{c_{1}} \ldots x_{m}^{i_{m}} \mid \varepsilon_{i}=0\right.$ or 1$\}$.

(1.3) $M \subset \operatorname{Im} i^{*}$ for the induced homomorphism $i^{*}: H^{*}\left(X ; Z_{p}\right) \longrightarrow$ $H^{*}\left(G ; Z_{p}\right)$.

(1.4) $P\left(H^{*}\left(X ; Z_{p}\right), t\right)=P(M, t) \cdot P\left(H^{*}\left(B ; Z_{p}\right), t\right) \cdot \prod_{i=1}^{m}\left(1-t^{\mathrm{deg} x_{i}+1}\right)$, where $P$ indicates the Poincaré series: $P\left(\sum V^{j}, t\right)=\sum\left(\operatorname{dim} V^{j}\right) t^{j}$.

The purpose of this section is to prove the following

Theorem 1.1. Let $N$ be a positive integer and assume that the principal bundle (1.1) satisfies (1.2), (1.3) and (1.4) for degree $\leq N$. Then, for a suitable choice of the elements $x_{i}$, the followings hold:

(i) For degree $\leq N-1, M=\operatorname{Im} i^{*}$ and the set of the transgressive elements is spanned by $M^{+}$and $\left\{x_{i} \mid \operatorname{deg} x_{i} \leq N-1\right\}$.

(ii) $\quad H^{*}\left(X ; Z_{p}\right)$ is isomorphic to $M \otimes \operatorname{Im} \pi^{*}$ as $\operatorname{Im} \pi^{*}$-modules for degree $\leq N$.

(iii) For transgression images $\left\{r_{i}\right\}$ of $\left\{x_{i} \mid \operatorname{deg} x_{i} \leq N-1\right\}$, we have a natural isomorphism $H^{*}\left(B ; Z_{p}\right) /\left(r_{i}\right) \cong \operatorname{Im} \pi^{*}$ for degree $\leq N$.

(iv) The elements $\left\{r_{i}\right\}$ are of no relation in $H^{*}\left(B ; Z_{p}\right)$ up to degree $N$.

Here we call that homogeneous elements $\left\{r_{i}\right\}$ of a graded commutative algebra $A$ over $Z_{p}$ are of no relation in $A$ up to degree $N$ if one of the following equivalent conditions holds (cf. [6]) :

(1.5), (i). The multiplication by $r_{i}$ is an injection of $A /\left(r_{1}, \ldots, r_{i-1}\right)$ 
in itself for degree $\leq N$.

(ii). There exists a submodule $B$ of $A$ such that the natural map of $Z_{p}\left[r_{1}, r_{2}, \ldots\right] \otimes B$ into $A$ is bijective for degree $\leq N$.

(iii). $P\left(A /\left(r_{1}, r_{2}, \ldots\right), t\right)=P(A, t) \cdot \prod_{i}\left(1-t^{\text {deg }{ }^{r_{i}}}\right)$ for degree $\leq N$.

Let $\left(E_{r}^{s, q}\right)$ be the $\bmod p$ cohomology spectral sequence associated with the principal bundle $(1.1)$, then

$E_{2}^{*, *}=H^{*}\left(B ; Z_{p}\right) \otimes H^{*}\left(G ; Z_{p}\right)$ converging to $H^{*}\left(X ; Z_{p}\right)$,

$\operatorname{Im} i^{*}=E_{\infty}^{0, *} \subset E_{2}^{0, *}=H^{*}\left(G ; Z_{p}\right)$ and $\operatorname{Im} \pi^{*}=E_{\infty}^{*, 0} \subset H^{*}\left(X ; Z_{p}\right)$.

Lemma 1. 1. (i). The multiplication gives an injection of $E_{r}^{*, 0} \otimes$ $E_{r}^{0, *}$ into $E_{r}^{* * *}$.

(ii). Let $\tilde{M}$ be a graded submodule of $H^{*}\left(X ; Z_{p}\right)$ which is injectively mapped into $H^{*}\left(G ; Z_{p}\right)$ under $i^{*}$, then the cup-product gives an injection of $\tilde{M} \otimes \operatorname{Im} \pi^{*}$ into $H^{*}\left(X ; Z_{p}\right)$.

Proof. The right translation $\mu$ gives a commutative diagram

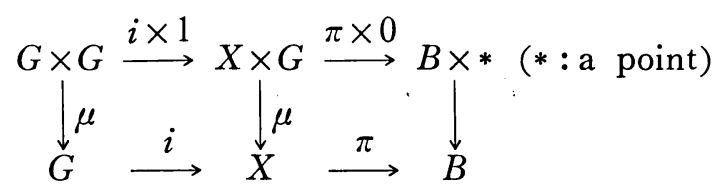

which is a map of fiberings, and induces a map of spectral sequences

$$
\mu^{*}: E_{r}^{s * *} \longrightarrow E_{r}^{s, *} \otimes H^{*}\left(G ; Z_{p}\right)
$$

such that $\mu^{*}(b)=b \otimes 1$ for $b \in E_{r}^{s, 0}$ and $\mu^{*}(z)=1 \otimes z+\sum z_{i}^{\prime} \otimes z_{i}^{\prime \prime}(\operatorname{deg}$ $\left.z_{i}^{\prime}>0\right)$ for $z \in E_{r}^{0, q} \subset E_{2}^{0, q}=H^{q}\left(G ; Z_{p}\right)$. Then $\mu^{*}(b \cdot z)=b \otimes z+\sum b \cdot z_{i}^{\prime}$ $\otimes z_{i}^{\prime \prime}$, and the assertion (i) is proved. (ii) is proved similarly by considering $\mu^{*}: H^{*}\left(X ; Z_{p}\right) \longrightarrow H^{*}\left(X ; Z_{p}\right) \otimes H^{*}\left(G ; Z_{p}\right)$ in which $\mu^{*}(x)=x \otimes 1$ holds for $x \in \operatorname{Im} \pi^{*}$.

q. e. d.

We assume the following inductive hypothesis for $n<N(\varepsilon=0,1)$. (1.6). The elements $x_{i}$ of $\operatorname{deg} x_{i} \leq n-1$ are transgressive and transgression images $\left\{r_{i}\right\}$ are of no relation up to degree $n+\varepsilon$.

Put $M_{n+1}=\sum_{j=0}^{n+1} M^{j}$ then $M_{n+1} \subset \operatorname{Im} i^{*}$ by the assumption 
of the theorem, and the differential $d_{r}$ of the spectral sequence satisfies $\begin{array}{lll} & d_{r}(b \otimes 1)=d_{r}(1 \bigotimes m)=0 & \text { for } b \in H^{*}\left(B ; Z_{p}\right) \text { and } m \in M_{n+1} \\ \text { and } & d_{r}\left(1 \otimes x_{i}\right)=0\left(r \leq \operatorname{deg} x_{i}\right), & d_{r}\left(1 \otimes x_{i}\right)=r_{i} \otimes 1 \quad\left(r=\operatorname{deg} x_{i}+1\right)\end{array}$ for $\operatorname{deg} x_{i} \leq n-1$. We put

$$
\begin{aligned}
& \Delta_{r}=\Delta\left(x_{i} ; r-1 \leq \operatorname{deg} x_{i} \leq n-1\right) \quad\left(\Delta(\phi)=Z_{p}\right) \\
& J_{r}=\text { the ideal of } H^{*}\left(B ; Z_{p}\right) \text { generated by }\left\{r_{i} \mid \operatorname{deg} r_{i}<\right. \\
& \operatorname{Min}(r, n+1)\}
\end{aligned}
$$

and $\quad \bar{E}_{r}^{*, *}=H^{*}\left(B ; Z_{p}\right) / J_{r} \otimes\left(\Delta_{r} \otimes M_{n+1}\right)$.

A differential $d_{r}$ in $\bar{E}_{r}^{*, *}$ is defined by the derivativity and the above equalities for $d_{r}$. Then using (1.5) we have easily

(1.7). $\bar{E}_{r+1}^{* * *} \subset H\left(\bar{E}_{r}^{*, *}\right)$ and the equality $\bar{E}_{,+1}^{s, q}=H\left(\bar{E}_{r}^{s, \theta}\right)$ holds if $q<$ $r-1$ or $r \geq n+1$ or if $s+r \leq n+\varepsilon$. Also $\left\{r_{i}\right\}$ are of no relation up to degree $k$ if and only if the equality $\bar{E}_{r+1}^{s, r-1}=H\left(\bar{E}_{r}^{s, r-1}\right)$ holds for $s+r$ $\leq k$.

Now we have natural maps

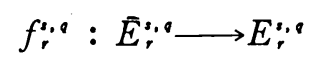

which commutes with the differential $d_{r}$ and induces

$$
\bar{f}_{r+1}^{s, q}: H\left(\bar{E}_{r}^{s, q}\right) \longrightarrow E_{r+1}^{s, q}=H\left(E_{r}^{s, q}\right) \text { such that } f_{r+1}^{s, q}=\bar{f}_{r+1}^{s, q} \mid \bar{E}_{r+1}^{s, q}
$$

Lemma 1.2. (1.6), implies the followings:

(i). $f_{i, \theta}^{*}$ is injective if $s \leq n+1+\varepsilon$ and $r \leq n+1$ or if $s \leq n$.

(ii). f; is surjective if $q=0$, if $s+q \leq n-1+\varepsilon$ and $q \leq n-1$ or if $s \leq n+1+\varepsilon-r$ and $q \leq n-1$.

(iii) The natural map of Coker $f_{r+1}^{0, n}$ into Coker $f_{r}^{0, n}$ is injective for $2 \leq r \leq n$.

(iv) Let $\varepsilon=0$ and $1 \leq q \leq n-1$, then $H\left(\bar{E}_{q+1}^{n-q, q}\right) / \bar{E}_{q+2}^{n-q . q}$ is isomorphic to Coker $f_{r}^{n-a, \theta}$ for $r \geq q+2$.

Proof. (i), and (ii), are obvious for $r=2$ and also for $s<0$ or $q<0 . \quad \bar{f}_{r+1}^{s, q}$ is injective (resp. surjective) if $f_{r}^{s, q}$ is injective (resp. surjective) and $f_{r}^{s-r, q+r-1}$ is surjective (resp. $f_{r}^{t+r, q-r+1}$ is injective). By induction on $r \geq 2$, the assertion (ii), by use of (1.7), the 
assertion (i), for $s=0$ or $q=0$, and then the assertion (i), by Lemma 1.1 since $\bar{E}_{r}^{:, q}=\bar{E}_{r}^{:, 0} \otimes \bar{E}_{r}^{0, q}$, are proved. Next we have the following commutative exact diagram

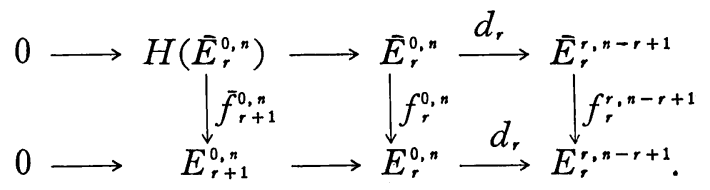

For $2 \leq r \leq n, H\left(\bar{E}_{r}^{0, n}\right)=\bar{E}_{r+1}^{0, n}$ by (1.7) and $f_{r}^{r, n-r+1}$ is injective by (i).. Then $\operatorname{Im} f_{r}^{0, n} \cap E_{r+1}^{0, n} \subset \operatorname{Im} \bar{f}_{r+1}^{0, n}=\operatorname{Im} f_{r+1}^{0, n}$, and (iii) follows.

Let $\varepsilon=0$ and $1 \leq q \leq n-1 . \quad \bar{f}_{q+2}^{n-q, q}$ is bijective since $f_{q+1}^{n-q, q}$ is bijective $f_{q+1}^{n-2 q-1,2 q}$ is surjective and $f_{q+1}^{n+1,0}$ is injective. Thus (iv) is true for $r=q+2$. Let $r>q+2$, then $H\left(\bar{E}_{r-1}^{n-q . q}\right)=\bar{E}_{r}^{n-q .8}$ by $(1.7)$ and we have an exact sequence $\stackrel{d_{r-1}}{\longrightarrow} \bar{E}_{r-1}^{n-q, q} \longrightarrow \bar{E}_{r}^{n-q, q} \longrightarrow 0$. The same holds for $\left\{E_{r}\right\}$. By the compatibility of $\left\{f_{r}\right\}$ we have an exact sequence

Coker $f_{r-1}^{n-q-r+1, q+r-2} \stackrel{d_{r-1}}{\longrightarrow}$ Coker $f_{r-1}^{n-q, q} \longrightarrow$ Coker $f_{r}^{n-q, q} \longrightarrow 0$.

The first cokernel is trivial by (ii). Therefore (iv) is proved by induction on $r \geq q+2$.

Lemma 1. 3. (1.6)。 implies (1.6) ${ }_{1}$ and that Coker $f_{n+1}^{0, n}$ is naturally isomorphic to Coker $f_{2}^{0, n}$ and it is mapped isomorphically onto $d_{n+1}\left(E_{n+1}^{0, n}\right)$ $\subset E_{n+1}^{n+1,0}$ under $d_{n+1}$.

Proof. We assume (1.6).. For degree $\leq n+\varepsilon, P(M, t)=\mathrm{P}\left(M_{n+1}\right.$, $t)=P\left(\bar{E}_{\infty}^{*, 0}, t\right)$ and $P\left(H^{*}\left(B ; Z_{p}\right), t\right) \cdot \prod_{i=1}^{m}\left(1-t^{\operatorname{deg} x_{i}+1}\right)=P\left(H^{*}\left(B ; Z_{p}\right) /\right.$ $\left.\left(r_{i}\right), t\right) \cdot\left(1-a_{n} t^{n+1}\right)=P\left(\bar{E}_{\infty}^{*, 0}, t\right) \cdot\left(1-a_{n} t^{n+1}\right)$ by $(1.5)$, where $a_{n}=\operatorname{dim}$ Coker $f_{2}^{0, n}=$ number of $\left\{x_{i} \mid \operatorname{deg} x_{i}=n\right\}$. Since $\bar{E}_{\infty}^{*, *}=\bar{E}_{\infty}^{*, 0} \otimes \bar{E}_{\infty}^{0, *}$, it follows from (1.4)

$$
\begin{gathered}
\text { (1. 8).. } P\left(E_{\infty}^{*, *}, t\right)=P\left(H^{*}\left(X ; Z_{p}\right), t\right)=P\left(\bar{E}_{\infty}^{*, *} t\right) \cdot\left(1-a_{n} t^{n+1}\right) \\
\text { for degree } \leq n+\varepsilon .
\end{gathered}
$$

By (i)o of Lemma 1.2, $f_{\infty}^{s, q}$ is injective, and $\operatorname{dim} E_{\infty}^{s, q} \geq \operatorname{dim} \bar{E}_{\infty}^{s, q}$ for $s \leq n$. By $(1.8)_{0}, \sum_{s+q=n} \operatorname{dim} E_{\infty}^{s, q}=\sum_{s+q=n} \operatorname{dim} \bar{E}_{\infty}^{s, q}$. Thus $f_{\infty}^{s, q}$ is 
bijective for $s+q=n$. By (iv) of Lemma 1.2, we have $H\left(\bar{E}_{q+1}^{s, q}\right)$ $=\bar{E}_{q+2}^{s, q}$ for $s+q=n\left(\bar{E}_{n+1}^{0, n}=\bar{E}_{n+2}^{0, n}=M^{n}\right)$. Thus (1.6)。 implies $(1.6)_{1}$ by use of (1.7) for $k=n+1$.

Next, assuming $(1.6)_{1}$ it follows from $(1.8)_{1}$

$$
a_{n}=\sum_{s+q=n+1}\left(\operatorname{dim} \bar{E}_{\infty}^{s, q}-\operatorname{dim} E_{\infty}^{s, q}\right) \leq \operatorname{dim} \bar{E}_{n+2}^{n+1,0}-\operatorname{dim} E_{n+2}^{n+1,0} .
$$

$\bar{E}_{n+2}^{n+1,0}=\bar{E}_{n+1}^{n+1,0}$ by definition. $\quad \bar{E}_{n+1}^{n+1,0}$ is isomorphic to $E_{n+1}^{n+1,0}$ by (i) and (ii) $)_{1}$ of Lemma 1.2. Since $d_{n+1}=0$ in $\bar{E}_{n+1}^{*, *}, d_{n+1}\left(\operatorname{Im} f_{n+1}^{0, n}\right)=$ $f_{n+1}^{n+1,0}\left(d_{n+1} \bar{E}_{n+1}^{0, n}\right)=0$ and $d_{n+1}$ induces a surjection of Coker $f_{n+1}^{0, n}$ onto $\operatorname{Im} d_{n+1} \subset E_{n+1}^{n+1,0}$. We have also $E_{n+2}^{n+1,0}=E_{n+1}^{n+1,0} / \operatorname{Im} d_{n+1}$. Thus

$$
\begin{aligned}
\operatorname{dim} \text { Coker } f_{2}^{0, n}=a_{n} & \leq \operatorname{dim} E_{n+1}^{n+1,0}-\operatorname{dim} E_{n+2}^{n+1,0} \\
& =\operatorname{dim} \operatorname{Im} d_{n+1} \leq \operatorname{dim} \text { Coker } f_{n+1}^{0, n} .
\end{aligned}
$$

By (iii) of Lemma 1.2, the equality $\operatorname{dim}$ Coker $f_{2}^{0, n}=\operatorname{dim} \operatorname{Im} d_{n+1}$ $=\operatorname{dim}$ Coker $f_{n+1}^{0, n}$ holds and the second half of the lemma is proved.

Proof of Theorem 1.1.

The theorem is obvious for $N=1$. By induction on $N$, we may assume (1.6)。 for $n=N-1$. Let $\left\{x_{k}\right\}$ be the set of $x_{i}$ with $\operatorname{deg} x_{i}=n$. By definition Coker $f_{2}^{0, n}$ has a basis $\left\{x_{k}\right\} \bmod \bar{E}_{2}^{0, *}=\Delta_{2}$ $\otimes M, \Delta_{2}=\Delta\left(x_{i} ; \operatorname{deg} x_{i}<n\right)$. By Lemma 1.3 there exist elements $x_{k}^{\prime}$ $\equiv x_{k} \bmod \Delta_{2} \otimes M$ such that $x_{k}^{\prime}\left(=1 \otimes x_{k}^{\prime}\right) \in E_{n+1}^{0, n}$ and that $\left\{x_{k}^{\prime}\right\}$ and $M^{n}=\bar{E}_{n+1}^{0, n}$ span $E_{n+1}^{0, n}$. Changing $x_{k}^{\prime}$ modulo $M^{n}$ if it is necessary, we may choose $x_{k}^{\prime}$ such that $x_{k}^{\prime \prime} \equiv x_{k} \bmod$ decomposables. Then replacing $x_{k}$ by $x_{k}^{\prime}$ we obtain new generators $\left\{x_{i}\right\}$ satisfying (1.2) and $x_{k} \in E_{n+1}^{0, n}$. Since $E_{n+1}^{0, n}$ coincides with the set of the transgressive elements of degree $n$, (i) of Theorem 1.1 is proved.

Let $r_{k}$ 's be transgression images of the $x_{k}$ 's. Lemma 1. 3 shows that $(1.6)_{1}$ holds and that $\left\{r_{k}\right\}$ are linearly independent in $E_{n+1}^{n+1.0}$ $\cong \bar{E}_{n+1}^{n+1.0} \subset H^{*}\left(B ; Z_{p}\right) /\left(r_{i} ; \operatorname{deg} r_{i} \leq n\right)$. Thus (iv) of Theorem 1.1 is proved. Again by Lemma 1.3

$$
\pi^{*} H^{n+1}\left(B ; Z_{p}\right)=E_{\infty}^{n+1,0}=E_{n+2}^{n+1,0} \cong E_{n+1}^{n+1,0} /\left\{r_{k}\right\},
$$


and (iii) follows. In Lemma 1.1, (ii), take $\tilde{M}$ such that it is mapped isomorphically onto $M_{n+1}$, then $\tilde{M} \otimes \operatorname{Im} \pi^{*}$ is mapped injetaively into $H^{*}\left(X ; Z_{p}\right)$. The Poincaré series of $H^{*}\left(X ; Z_{p}\right)$ and $M \otimes \operatorname{Im} \pi^{*}$ are given both sides of (1.4) for degree $\leq n+1$. Thus (ii) of the theorem is proved.

q.e.d.

\section{§. Cohomology of some homogeneous spaces.}

Let $U$ be a connected subgroup of $G$ and let $E G \longrightarrow B U=E G$ $/ U$ be a universal $U$-bundle. In the principal $G$-bundle

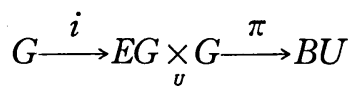

the projection $E G \underset{v}{\times} G \longrightarrow G / U=* \underset{v}{\times} G$ is a homotopy equivalence. So we have a fibering

$$
G \stackrel{\pi_{0}}{\longrightarrow} G / U \stackrel{i_{0}}{\longrightarrow} B U
$$

equivalent to (2.1), where $i_{0}$ is a map classifying the $U$-bundle : $G \longrightarrow G / U$.

By Hopf-Borel theorem [6], we have for each prime $p$

$$
H^{*}\left(G ; Z_{p}\right)=\Lambda\left(x_{1}^{\prime}, \ldots, x_{r^{\prime}}^{\prime}\right) \otimes Z_{p}\left[y_{1}^{\prime}, \ldots, y_{s^{\prime}}^{\prime}\right] /\left(y_{1}^{h_{1}}, \ldots, y_{s^{\prime}}^{h_{s^{\prime}}}\right)
$$

where $h_{j}$ is a power of $p\left(h_{j} \geq 4\right.$ if $\left.p=2\right)$ and if $p>2$ then $\operatorname{deg} x_{i}^{\prime}$ is odd and $\operatorname{deg} y_{j}^{\prime}$ is even, and for the rational coefficient

$$
H^{*}(G ; Q)=\Lambda\left(z_{1}, \ldots, z_{\ell}\right), \quad \operatorname{deg} z_{i}: o d d .
$$

Let $M$ be the subalgebra generated by $\left\{y_{j}^{\prime} \mid \operatorname{deg} y_{j}^{\prime}\right.$ even $\}$ and additionally $\left\{y_{j}^{\prime 2}\right\}$ and $\left\{x_{i}^{\prime} \mid \operatorname{deg} x_{i}^{\prime}\right.$ even $\}$ if $p=2$, and let $\left\{x_{i} \mid 1 \leq i \leq\right.$ $r\}$ be the union of $\left\{x_{i}^{\prime} \mid \operatorname{deg} x_{i}^{\prime}\right.$ odd $\}$ and $\left\{y_{j}^{\prime} \mid \operatorname{deg} y_{j}^{\prime} \operatorname{odd}(p=2)\right\}$. Then (1.2) is satisfied:

$$
\Delta\left(x_{1}, \ldots, x_{r}\right) \otimes M \cong H^{*}\left(G ; Z_{p}\right)
$$

where $\quad M=Z_{p}\left[y_{1}, \ldots, y_{s}\right] /\left(y_{1}^{k_{1}}, \ldots, y_{s}^{k_{s}}\right) \quad$ with $k_{j}:$ power of $p$.

Now we consider the following hypothesis (2. 6). $r=\ell$ and for each $j, 1 \leq j \leq s$, there corresponds an $i=i(j)$ such 
that $x_{i(j)}$ is transgressive with respect to (2.2) and that $\beta\left(x_{i(j)}\right)=y_{j}$, where $\beta$ indicates the Bockstein homomorphism assoicated with the exact sequence $0 \longrightarrow Z_{p} \longrightarrow Z_{p^{2}} \longrightarrow Z_{p} \longrightarrow 0$.

We shall see in $\S 3$ that the simply connected simple Lie groups $G$ enjoy (2.6) if $U=T$ or if $(G, p) \neq\left(E_{8}, 2\right)$.

We shall consider the case that $U$ is torsion free and of maximal rank. According to Borel [6],

and

$$
H^{*}(U)=\Lambda\left(u_{1}, \ldots, u_{\ell}\right), \quad \operatorname{deg} u_{i}: \operatorname{odd},
$$

$$
H^{*}(B U)=Z\left[t_{1}, \ldots, t_{\ell}\right], \quad \operatorname{deg} t_{i}=\operatorname{deg} u_{i}+1: \text { even, }
$$

where $t_{i}$ is a transgression image of $u_{i}$. We shall denote the $i_{0}^{*}-$ image of $t_{i}$ by the same symbol

$$
t_{i}=i_{0}^{*}\left(t_{i}\right) \ni H^{*}(G / U) .
$$

Assuming (2.6) for all prime $p$, we denote $\left\{y_{1}, \ldots, y_{m}\right\}$ the collection of the $y_{j}$ in (2.6) for all possible prime, and by $p_{j}$ the prime corresponding to $y_{j}$.

Then we have the following description of $H^{*}(G / U)$.

Theorem 2. 1. Let $U$ be a torsion free connected subgroup of maximal rank in $G$. Assume (2.6) for all prime, and let $\delta_{j}$ and $\sigma_{i}$ be homogeneous elements of $Z\left[t_{1}, \ldots, t_{\ell}\right]$ such that $\delta_{j}\left(\bmod p_{j}\right)$ is a transgression image of $x_{i(j)}$ and that $H^{*}(G / U ; Q)=Q\left[t_{1}, \ldots, t_{\ell}\right] /\left(\sigma_{1}, \ldots, \sigma_{\ell}\right)$, $\operatorname{deg} \sigma_{1} \leq \ldots \leq \operatorname{deg} \sigma_{\ell}$.

Then there exist generators $\gamma_{j} \in H^{*}(G / U)$ and relations $\rho_{i}, \rho_{j}^{\prime} \in Z$ $\left[t_{1}, \ldots, t_{\ell}, \gamma_{1}, \ldots, \gamma_{m}\right]$ such that $\left(\operatorname{deg} \rho_{i}=\operatorname{deg} \sigma_{i}, \operatorname{deg} \gamma_{j}=\operatorname{deg} \rho_{j}^{\prime}=\operatorname{deg}\right.$ $\left.\delta_{j}\right)$

$$
\begin{aligned}
& H^{*}(G / U)=Z\left[t_{1}, \ldots, t_{\ell}, \gamma_{1}, \ldots, \gamma_{m}\right] /\left(\rho_{1}, \ldots, \rho_{\ell}, \rho_{1}^{\prime}, \ldots, \rho_{m}^{\prime}\right), \\
& \pi_{0}^{*}\left(\gamma_{j}\right) \equiv y_{j} \quad\left(\bmod p_{j}\right)
\end{aligned}
$$

and $\quad \rho_{j}^{\prime}=p_{j} \cdot \gamma_{j}+\delta_{j}$,

where the relation $\rho_{i}$ is determined by the maximality of the integer $n$ in 


$$
n \bullet \rho_{i} \equiv \sigma_{i} \quad \bmod \left(\rho_{1}, \ldots, \rho_{i-1}, \rho_{1}^{\prime}, \ldots, \rho_{m}^{\prime}\right) .
$$

In order to prove the theorem we prepare two lemmas. The first lemma is well-known and proved by checking integral cochains.

Lemma 2. 1. Let $F \stackrel{i}{\longrightarrow} X \stackrel{\pi}{\longrightarrow} B$ be a fibering, $x$ a transgressive element of $H^{n}\left(F ; Z_{p}\right)$ and let $\delta$ be an element of $H^{n+1}(B)$ such that $\delta(\bmod p)$ is a transgression image of $x$. Then there exists an element $\gamma$ of $H^{n+1}(X)$ such that

$$
i^{*}(\gamma) \equiv \beta(x)(\bmod p) \text { and } p \cdot \gamma=-\pi^{*}(\delta) .
$$

The rational cohomology ring $H^{*}(G / U ; Q)$ is determined by the action of the Weyl groups $\Phi(G)$ and $\Phi(U)$ on a maximal torus $T \subset U \subset G[6: C h . V I]$ :

$$
\text { (2. 7) } \quad \begin{aligned}
H^{*}(B G ; Q) & =H^{*}(B T ; Q)^{\bullet(G)} \subset H^{*}(B U ; Q)=H^{*}(B T ; Q)^{\oplus(U)} \\
\text { and } \quad & \quad \\
H^{*}(G / U ; Q) & =H^{*}(B U ; Q) /\left(H^{+}(B G ; Q)\right) \\
& =Q\left[t_{1}, . ., t_{\ell}\right] /\left(\sigma_{1}, . ., \sigma_{\ell}\right)
\end{aligned}
$$

where $\sigma_{i}$ is a transgression image of $z_{i}$ and $\left\{\sigma_{i}\right\}$ are of no relation in $H^{*}(B U ; Q)=Q\left[t_{1}, \ldots, t_{\ell}\right]$. By $[10], G / T$ and $U / T$ are torsion free. Since $U$ is torsion free, so is $G / U$ by Proposition 30.1 of [6]. Thus

$$
P\left(H^{*}\left(G / U ; Z_{p}\right), t\right)=P\left(H^{*}\left(B U ; Z_{p}\right), t\right) \cdot \prod_{i=1}^{\ell}\left(1-t^{\operatorname{deg} z_{i}+1}\right) .
$$

Lemma 2. 2. The assumption of Theorem 2. 1 implies the assumptions (1.2), (1.3), (1.4) of Theorem 1.1 for all prime and for arbitrary $N$.

Proof. (1.2) is already satisfied by (2.5). Since $x_{i(j)}$ is transgressive, so is $y_{j}=\beta\left(x_{i(j)}\right)$. Since $H^{n}\left(B U ; Z_{p}\right)=0$ for odd $n$, the transgression image of $y_{j}$ is trivial, that is, $y_{j}$ is an $i_{0}^{*}$-image. It follows (1.3) $: M \subset \operatorname{Im} i_{0}^{*}$. Consider the $\bmod p$ Bockstein spectral sequence $\left(E_{r}\right)$ for $G: E_{1}=H^{*}\left(G ; Z_{p}\right), E_{2}=H\left(E_{1}\right.$ w. r. t. $\left.\beta\right)$ converging to $E_{\infty}=\left(H^{*}(G) /\right.$ torsion $) \otimes Z_{p}=\Lambda\left(z_{1}, \ldots, z_{\ell}\right)$. From (2.6) we 
have $E_{2}$ as a cohomology (subquotient) of

$$
E_{2}^{\prime}=\Delta\left(x_{i}(i \neq i(j) \text { for any } j), x_{i(j)} y_{j}^{k_{j}-1}\right) .
$$

Then $\operatorname{dim} E_{2} \leq \operatorname{dim} E_{2}^{\prime}=2^{\ell}$, but $2^{\ell}=\operatorname{dim} E_{\infty} \leq \operatorname{dim} E_{2}$. Thus we have $E_{2}^{\prime}=E_{\infty}$ and

(2.9). The set $\left\{\operatorname{deg} z_{i}\right\}$ coincides with the set

$\left\{\operatorname{deg} x_{i}(i \neq i(j)), k_{j} \cdot \operatorname{deg} y_{j}-1\right\}$.

That is, $\prod_{i=1}^{\ell}\left(1-t^{\operatorname{deg} x_{i}+1}\right)=\prod_{i \neq i(j)}\left(1-t^{\mathrm{deg} x_{i}+1}\right) \cdot \prod_{j=1}^{j}\left(1-t^{h_{j} \cdot \operatorname{deg} y_{j}}\right)=\prod_{i=1}^{\ell}(1-$ $\left.t^{\mathrm{deg} x_{i}+1}\right) \cdot P(M, t)$. Then it follows from (2.8) that (1.4) holds:

$$
P\left(H^{*}\left(G / U ; Z_{p}\right), t\right)=P\left(H^{*}\left(B U ; Z_{p}\right), t\right) \cdot P(M, t) \cdot \prod_{i=1}^{\ell}\left(1-t^{\mathrm{deg} s_{i}+1}\right) .
$$

Proof of Theorem 2.1. Apply Lemma 2. 1 to $x=x_{i(j)}, p=p_{i}$ and $\delta=\delta_{j}$, then we have the existence of $\gamma_{j}$ such that $\pi_{0}^{*}\left(\gamma_{j}\right) \equiv y_{j}\left(\bmod p_{j}\right)$ and that $\rho_{j}^{\prime}=p_{j} \cdot \gamma_{j}+\delta_{j}$ vanishes in $H^{*}(G / U)$. Put

$$
R=Z\left[t_{1}, \ldots, t_{\ell}, \gamma_{1}, \ldots, \gamma_{m}\right] \text { and } I_{i}=\left(\rho_{1}, \ldots, \rho_{i}, \rho_{1}^{\prime}, \ldots, \rho_{m}^{\prime}\right) \subset R .
$$

Since $\sigma_{i} \neq 0$ in $\left(R / I_{i-1}\right) \otimes Q=H^{*}(B U ; Q) /\left(\sigma_{1}, \ldots, \sigma_{i-1}\right), \sigma_{i}\left(\bmod I_{i-1}\right)$ is of infinite order. Since $R / I_{i-1}$ is finitely generated for each degree, there exists the maximum of the integers $n$ such that $n \cdot x \equiv \sigma_{i}\left(\bmod I_{i-1}\right)$ for some $x$. So, the existence of the relation $\rho_{i}$ with the required property is proved inductiely. We have obtained a natural homomorphism $\eta: R / I_{\ell} \longrightarrow H^{*}(G / U)$, and by tensoring $Z_{p}, \eta_{p}:\left(R / I_{\ell}\right) \otimes Z_{p} \longrightarrow H^{*}\left(G / U ; Z_{p}\right)$. Then it is sufficient to prove that $\eta_{p}$ is bijective for each prime $p$.

By Lemma 2.2 we apply Theorem 1.1 to (2.2), and obtain $H^{*}\left(G / U ; Z_{p}\right)=R_{p} /\left(r_{1}, \ldots, r_{\ell}, r_{1}^{\prime}, \ldots, r_{s}^{\prime}\right)$ for $R_{p}=Z_{p}\left[t_{1}, \ldots, t_{\ell}, \gamma_{1}, \ldots, \gamma_{s}\right]$, where $r_{j}^{\prime}$ is a relation satisfying $r_{j}^{\prime} \equiv \gamma_{j}^{h_{j}} \bmod \left(t_{1}, \ldots, t_{\ell}\right)$. On the other hand, in $\left(R / I_{\ell}\right) \otimes Z_{p}, \gamma_{j}$ and $\delta_{j}$ are cancelled to each other if $p_{j} \neq p$, and $\rho_{j}^{\prime}$ is replaced by $\delta_{j}=r_{i(j)}$ if $p_{j}=p$. Thus $\eta_{p}$ is equivalent to the natural map

$$
R_{p} /\left(\rho_{1}, \ldots, \rho_{\ell}, \delta_{1}, \ldots, \delta_{s}\right) \longrightarrow R_{p} /\left(r_{1}^{\prime \prime}, \ldots, r_{\ell}^{\prime \prime}, \delta_{1}, \ldots, \delta_{s}\right),
$$

where $\left\{r_{i}^{\prime \prime}\right\}=\left\{r_{i} \mid i \neq i(j)\right\} \cup\left\{r_{1}^{\prime}, \ldots, r_{s}^{\prime}\right\}$. By (2.9), we may assume 
that $\operatorname{deg} \rho_{i}=\operatorname{deg} r_{i}^{\prime \prime} . \quad$ Put

$$
J_{i}=\left(\rho_{1}, \ldots, \rho_{i}, \delta_{1}, \ldots, \delta_{s}\right) \text { and } J_{i}^{\prime \prime}=\left(r_{1}^{\prime \prime}, \ldots, r_{i}^{\prime \prime}, \delta_{1}, \ldots, \delta_{s}\right) \text {. }
$$

The property of $\rho_{i}$ shows that $\left\{\rho_{i+1}, \ldots, \rho_{\ell}\right\}$ are linearly independent $\bmod J_{i}$, and the same is true for $\left\{r_{i+1}^{\prime \prime}, \ldots, r_{\ell}^{\prime \prime}\right\} \bmod J_{i}^{\prime \prime}$. By induction on the degree of $\rho_{i}$ we assume that $J_{i}=J_{i}^{\prime \prime}$ for $\operatorname{deg} \rho_{i}<$ $\operatorname{deg} \rho_{i+1}=\ldots=\operatorname{deg} \rho_{k}<\operatorname{deg} \rho_{k+1}$. Then the equality $J_{k}=J_{k}^{\prime \prime}$ is proved at first for degree $\leq \operatorname{deg} \rho_{k}$ and then for all degree. So, we obtain $J_{\ell}=J_{\ell}^{\prime \prime}$, that is, $\eta_{p}$ is bijective and so is $\eta$.

q. e. d.

Corollary 2. 2. Theorem 2.1 gives a minimum system of generators and relations for $H^{*}(G / U)$ if there is no pair $(i, j)$ with $\operatorname{deg} t_{i}=\operatorname{deg} \rho_{j}$, $\operatorname{deg} t_{i}=\operatorname{deg} \rho_{j}^{\prime}$ or with $\operatorname{deg} \rho_{i}^{\prime}=\operatorname{deg} \rho_{j}^{\prime}$ and $p_{i} \neq p_{j}$.

\section{§3. $\mathbf{H}^{*}(\mathbf{G} / \mathbf{T})$ for simple Lie group $\mathbf{G}$.}

Let $G$ be a compact connected semi-simple Lie group and let $T$ be its maximal torus, then the universal covering $\tilde{G}$ of $G$ is compact and

$$
\tilde{G} / \tilde{T}=G / T
$$

for the inverse image $\tilde{T}$ of $T$ which is a maximal torus of $\tilde{G}$. By Corollary 2.2,

(3.2) if a simply connected compact $G$ satisfies (2.6) and if there is no pair $\left(y_{j}(\bmod p), y_{k}\left(\bmod p^{\prime}\right)\right)$ with $\operatorname{deg} y_{j}=\operatorname{deg} y_{k}$ and $p \neq p^{\prime}$, then Theorem 2.1 gives a minimum system of generators and relations for $H^{*}(G / T)$.

For classical cases we have

Proposition 3. 1. For $G=S U(\ell+1), S p(\ell)$ and $S O(n), S p i n(n)$, $\ell=\left[\begin{array}{l}n \\ 2\end{array}\right],(2.6)$ is satisfied for arbitrary $U$, and we have the following description of $H^{*}(G / U)$ by minimum systems of generators and relations :

$$
H^{*}(S U(\ell+1) / T)=Z\left[t_{1}, \ldots, t_{\ell}\right] /\left(\rho_{2}, \rho_{3}, \ldots, \rho_{\ell+1}\right),
$$




$$
H^{*}(S p(\ell) / T)=Z\left[t_{1}, \ldots, t_{\ell}\right] /\left(\rho_{2}, \rho_{4}, \ldots, \rho_{2 \ell}\right),
$$

and $\quad H^{*}(S O(n) / T)=H^{*}(\operatorname{Spin}(n) / \tilde{T})$

$$
=Z\left[t_{1}, \ldots, t_{\ell}, \gamma_{1}, \ldots, \gamma_{m}\right] /\left(\rho_{2}, \rho_{3}, \ldots, \rho_{\ell}, \rho_{2 m+2}^{\prime}, \rho_{2 m+4}, \ldots, \rho_{2 \ell}\right),
$$

where $\operatorname{deg} t_{i}=2, \operatorname{deg} \rho_{k}=\operatorname{deg} \rho_{k}^{\prime}=2 k, \operatorname{deg} \gamma_{j}=4 j+2, \quad m=\left[\frac{n-3}{4}\right]$ and $s=\left[\frac{n-1}{2}\right]$. (Explicit forms of the relations may be obtained from the results in $\$ 2$ of [12].)

Proof. Except the case $G=S O(n), S p i n(n)$ and $p=2, H^{*}\left(G ; Z_{p}\right)$ $=\Lambda\left(x_{1}, \ldots, x_{\ell}\right)$ and $(2.6)$ is satisfied. Let $z_{i} \in H^{i}\left(S O(n) ; Z_{2}\right)$ be the suspension image of the $(i+1)$-th Stiefel-Whitney class $w_{i+1}$ $\in H^{i+1}\left(B S O(n) ; Z_{2}\right)$. Then $z_{i}$ is universally transgressive and we have $z_{i}^{2}=z_{2 i}(=0$ if $2 i \geq n)$ and $\beta\left(z_{2 j-1}\right)=S q^{1}\left(z_{2 j-1}\right)=z_{2 j}$ by $\mathrm{Wu}$ formula. Thus (2.5) and (2.6) are satisfied for $x_{i}=z_{2 i-1}, y_{j}=z_{4 j-2}$ and for powers $h_{j}$ of 2 such that $n \leq h_{j}(4 j-2)<2 n$ :

$H^{*}\left(S O(n) ; Z_{2}\right)=\Delta\left(x_{1}, \ldots, x_{\ell}\right) \otimes M, \quad M=Z_{2}\left[y_{1}, \ldots, y_{s}\right] /\left(y_{1}^{h_{1}}, \ldots, y_{s}^{h_{s}}\right)$ for $s=\left[\frac{n+1}{4}\right]$. The covering homomorphism $p^{*}: H^{*}\left(S O(n) ; Z_{2}\right)$ $\longrightarrow H^{*}\left(\operatorname{Spin}(n) ; Z_{2}\right)$ has the kernel $\left(x_{1}, y_{1}\right)=\left(x_{1}\right)$ and $H^{*}($ Spin $\left.(n) ; Z_{2}\right)=\Delta(t) \otimes \operatorname{Im} p^{*}, \operatorname{deg} t=2 \cdot h_{1}-1$. Thus (2.6) is satisfied by omitting $y_{1}$ and by replacing $x_{1}$ by $t$. Then the above descriptions are obtained directly from Theorem 2.1, (2.9) and (3.2).

q. e. d.

The simply connected exceptional Lie groups have p-torsions only in the cases listed below [2], [3], [4], [5], [8], $\left(\operatorname{deg} x_{i}=i\right)$ :

$$
\begin{aligned}
H^{*}\left(G_{2} ; Z_{2}\right) & =\Delta\left(x_{3}, x_{5}\right) \otimes Z_{2}\left[x_{6}\right] /\left(x_{6}^{2}\right), \\
H^{*}\left(F_{4} ; Z_{2}\right) & =\Delta\left(x_{3}, x_{5}, x_{15}, x_{23}\right) \otimes Z_{2}\left[x_{6}\right] /\left(x_{6}^{2}\right), \\
H^{*}\left(E_{6} ; Z_{2}\right) & =\Delta\left(x_{3}, x_{5}, x_{9}, x_{15}, x_{17}, x_{23}\right) \otimes Z_{2}\left[x_{6}\right] /\left(x_{6}^{2}\right), \\
H^{*}\left(E_{7} ; Z_{2}\right) & =\Delta\left(x_{3}, x_{5}, x_{9}, x_{15}, x_{17}, x_{23}, x_{27}\right) \\
\otimes & Z_{2}\left[x_{6}, x_{10}, x_{18}\right] /\left(x_{6}^{2}, x_{10}^{2}, x_{18}^{2}\right), \\
H^{*}\left(E_{8} ; Z_{2}\right) & =\Delta\left(x_{3}, x_{5}, x_{9}, x_{15}, x_{17}, x_{23}, x_{27}, x_{29}\right) \\
\otimes & Z_{2}\left[x_{6}, x_{10}, x_{18}, x_{30}\right] /\left(x_{6}^{8}, x_{10}^{4}, x_{18}^{2}, x_{30}^{2}\right),
\end{aligned}
$$

and 
On the cohomology ring of some homogeneous spaces

where $x_{i+2}=S q^{2} x_{i}$ for $i=3,27 ; x_{i+4}=S q^{4} x_{i}$ for $i=5,25 ; x_{i+8}=S q^{8} x_{i}$ for $i=9,15$ and $x_{2 i}=x_{i}^{2}=\beta x_{2 i-1}$ for $i=3,5,9,15$.

$$
\begin{aligned}
& H^{*}\left(F_{4} ; Z_{3}\right)=\Lambda\left(x_{3}, x_{7}, x_{11}, x_{15}\right) \otimes Z_{3}\left[x_{8}\right] /\left(x_{8}^{3}\right), \\
& H^{*}\left(E_{6} ; Z_{3}\right)=\Lambda\left(x_{3}, x_{7}, x_{9}, x_{11}, x_{15}, x_{17}\right) \otimes Z_{3}\left[x_{8}\right] /\left(x_{8}^{3}\right), \\
& H^{*}\left(E_{7} ; Z_{3}\right)=\Lambda\left(x_{3}, x_{7}, x_{11}, x_{15}, x_{19}, x_{27}, x_{35}\right) \otimes Z_{3}\left[x_{8}\right] /\left(x_{8}^{3}\right),
\end{aligned}
$$

and

$$
H^{*}\left(E_{8} ; Z_{3}\right)=\Lambda\left(x_{3}, x_{7}, x_{15}, x_{19}, x_{27}, x_{35}, x_{39}, x_{47}\right)
$$

$$
\otimes Z_{3}\left[x_{8}, x_{20}\right] /\left(x_{8}^{3}, x_{20}^{3}\right),
$$

where $x_{7}=\mathscr{P}^{1} x_{3}, x_{8}=\beta x_{7}$ and $x_{20}=\beta x_{19}=\beta \mathscr{P}^{3} x_{11}$.

$$
H^{*}\left(E_{8} ; Z_{5}\right)=\Lambda\left(x_{3}, x_{11}, x_{15}, x_{23}, x_{27}, x_{35}, x_{39}, x_{47}\right) \text {. }
$$

$\otimes Z_{5}\left[x_{12}\right] /\left(x_{12}^{5}\right)$,

where $x_{11}=\mathscr{P}^{1} x_{3}$ and $x_{12}=\beta x_{11}$.

Proposition 3. 2. Let $p$ be a prime and $G$ a simply connected exceptional Lie group. Let $U$ be a connected subgroup of maximal rank in $G$ which is torsion free if $G=E_{8}$ and $p=2$. Then (2.6) is satisfied. $H^{*}(G / T)$ has the following minimum systems of generators and relations:

$$
\begin{aligned}
& H^{*}\left(G_{2} / T\right)=Z\left[t_{1}, t_{2}, \gamma_{3}\right] /\left(\rho_{2}, \rho_{3}, \rho_{6}\right), \\
& H^{*}\left(F_{4} / T\right)=Z\left[t_{1}, t_{2}, t_{3}, t_{4}, \gamma_{3}, \gamma_{4}\right] /\left(\rho_{2}, \rho_{3}, \rho_{4}, \rho_{6}, \rho_{8}, \rho_{12}\right) \\
& H^{*}\left(E_{6} / T\right)=Z\left[t_{1}, \ldots, t_{6}, \gamma_{3}, \gamma_{4}\right] /\left(\rho_{2}, \rho_{3}, \rho_{4}, \rho_{5}, \rho_{6}, \rho_{8}, \rho_{9}, \rho_{12}\right), \\
& H^{*}\left(E_{7} / T\right)=Z\left[t_{1}, \ldots, t_{7}, \gamma_{3}, \gamma_{4}, \gamma_{5}, \gamma_{9}\right] /
\end{aligned}
$$

$\left(\rho_{2}, \rho_{3}, \rho_{4}, \rho_{5}, \rho_{6}, \rho_{8}, \rho_{9}, \rho_{10}, \rho_{12}, \rho_{14}, \rho_{18}\right)$

and

$$
H^{*}\left(E_{8} / T\right)=Z\left[t_{1}, \ldots, t_{8}, \gamma_{3}, \gamma_{4}, \gamma_{5}, \gamma_{6}, \gamma_{9}, \gamma_{10}, \gamma_{15}\right] /
$$

$\left(\rho_{2}, \rho_{3}, \rho_{4}, \rho_{5}, \rho_{6}, \rho_{8}, \rho_{9}, \rho_{10}, \rho_{12}, \rho_{14}, \rho_{15}, \rho_{18}, \rho_{20}, \rho_{24}, \rho_{30}\right)$,

where $\operatorname{deg} t_{i}=2, \operatorname{deg} \gamma_{j}=2 j, \operatorname{deg} \rho_{h}=2 k$,

and $\quad \rho_{i}=2 \cdot \gamma_{i}+\delta_{i} \quad\left(i=3,5,9,15 ; \delta_{3} \equiv S q^{2} \rho_{2}, \delta_{5} \equiv S q^{4} \delta_{3}, \delta_{9} \equiv S q^{8} \delta_{5}\right.$,

$\left.\delta_{15} \equiv S q^{14} \rho_{8}(\bmod 2)\right), \quad \rho_{i}=3 \cdot \gamma_{i}+\delta_{i} \quad\left(i=4,10 ; \delta_{4} \equiv \mathscr{P}^{1} \rho_{2}\right.$,

$\left.\delta_{10} \equiv \mathscr{P}^{3} \delta_{4}(\bmod 3)\right)$ and $\rho_{6}=5 \cdot \gamma_{6}+\delta_{6}\left(\delta_{6} \equiv \mathscr{P}^{1} \rho_{2}(\bmod 5)\right)$. 
(For $G=G_{2}, F_{4}, E_{6}$ explicit forms of the relations may be obtained from the results of [12].)

Proof. Except $x_{30}(p=2)$, each generators $x_{2 i}$ of even degree satisfy $x_{2 i}=\beta x_{2 i-1}$ and $x_{2 i-1}=\alpha \cdot x_{3}$ for a cohomology operation $\alpha$. Since $x_{3}$ is universally transgressive, so is $x_{2 i-1}$ if $i \neq 15$. Thus (2.6) holds for $(G, p) \neq\left(E_{8}, 2\right)$. Now let $(G, p)=\left(E_{8}, 2\right)$ and $U$ be torsion free. Then Lemma 2.2 and thus Theorem 2.1 are valid for degree $\leq 29$. It follows from Theorem 1.1, (i) that there exists a transgressive element $x_{15}^{\prime} \equiv x_{15}$ mod decomposables. Putting $x_{15}^{\prime}=x_{15}$ $+a \cdot x_{9} x_{3}^{2}+b \cdot x_{5}^{3}+c \cdot x_{3}^{5} \quad\left(\mathrm{a}, \mathrm{b}, \mathrm{c} \in Z_{2}\right)$ we have $\left(x_{15}^{\prime}\right)^{4}=x_{15}^{4}+a \cdot x_{18}^{2} x_{6}^{4}+b \cdot x_{10}^{6}$ $+c \cdot x_{6}^{10}=0$. Thus we may replace $x_{15}$ by $x_{15}^{\prime}$ (so $x_{23}$ by $S q^{8} x_{15}^{\prime}$ and so on) in the last formula of (3.3). Then (2.6) is satisfied for torsion free $U$. By Theorem 2.1 and (3.2), we have the above descriptions of $H^{*}(G / T)$.

q. e. d.

Corollay 3. 3. For a suitable choice of the generators in (3.3), (3.4), (3.5), the generators are transgressive and satitfy (i) of Theorem 1.1 with respect to the fibering (2.2) for torsion free connected subgroup $U$ of maximal rank.

\section{DePARTMENT OF MATHEMATICS, KYOTO UNIVERSiTy}

\section{References.}

[1] S. Araki, On the non-commutativity of Pontrjagin rings mod 3 of some compact exceptional groups, Nagoya Math. J., 17 (1960), 225-260.

[2] S. Araki, Differential Hopf algebras and the cohomology mod 3 of the compact exceptional groups $\mathrm{E}_{7}$ and $\mathrm{E}_{8}$, Ann. of Math., 73 (1961), 404-436.

[3] S. Araki, Cohomology modulo 2 of the compact exceptional groups $\mathrm{E}_{6}$ and $\mathrm{E}_{7}, \mathrm{~J}$. Math. Osaka City U., 12 (1961), 43-65.

[4] S Araki, On cohomology mod $p$ of compact exceptional Lie groups (Japanese), Sugaku, 14-4 (1963), 219-235.

[5] S. Araki and Y. Shikata, Cohomology mod 2 of the exceptional group $\mathrm{E}_{8}$, Proc. Japan Acad., 37 (1961), 619-622.

[6] A. Borel, Sur la cohomologie des espaces fibrés principaux et des espaces homogènes de groups de Lie compacts, Ann. of Math., 57 (1954), 115-207.

[7] A. Borel, La cohomologie mod 2 de certains espaces homogènes, Comm. Math. Helv., 27 (1953), 165-197. 
[8] A. Borel, Sur l'homologie et la cohomologie des groupes de Lie compacts connexes, Amer. J. Math., 76 (1954), 273-342.

[9] A. Borel, Sous-groupes commutatifs et torsions des groupes de Lie compacts connexes, Tôhoku Math. J., 13 (1961), 216-240.

[10] R. Bott, An application of the Morse theory to the topology of Lie groups, Bull. Soc. Math. France, 84 (1956), 251-282.

[11] R. Bott and H. Samelson, Applications of the Morse theory to symmetric spaces, Amer. J. Math., 80 (1958), 964-1029.

[12] H. Toda and T. Watanabe, The integral cohomology ring of $F_{4} / T$ and $E_{6} / T$, J. Math. Kyoto U., 14 (1974), 257-286. 